\title{
High-quality high-order harmonic generation through pre-plasma truncation
}

\author{
B. Y. Li, ${ }^{1,2}$ F. Liu,${ }^{1,2}$ M. Chen,,${ }^{1,2, *}$ Z. Y. Chen,${ }^{3}$ X. H. Yuan, ${ }^{1,2}$ S. M. Weng,,${ }^{1,2}$ \\ T. Jin, ${ }^{4}$ S. G. Rykovanov, ${ }^{5}$ J. W. Wang, ${ }^{6}$ Z. M. Sheng, ${ }^{1,2,7,8, \dagger}$ and J. Zhang ${ }^{1,2}$ \\ ${ }^{1}$ Key Laboratory for Laser Plasmas (MoE), School of Physics and Astronomy, \\ Shanghai Jiao Tong University, Shanghai 200240, China \\ ${ }^{2}$ Collaborative Innovation Center of IFSA (CICIFSA), \\ Shanghai Jiao Tong University, Shanghai 200240, China \\ ${ }^{3}$ National Key Laboratory of Shock Wave and Detonation Physics, \\ Institute of Fluid Physics, China Academy of Engineering Physics, Mianyang 621999, China \\ ${ }^{4}$ Zhiyuan College, Shanghai Jiao Tong University, Shanghai 200240, China \\ ${ }^{5}$ Center for Computational and Data-Intensive Science and Engineering, \\ Skolkovo Institute of Science and Technology, Moscow 121205, Russia \\ ${ }^{6}$ Shanghai Institute of Optics and Fine Mechanics, \\ Chinese Academy of Sciences, Shanghai 201800, China \\ ${ }^{7}$ Tsung-Dao Lee Institute, Shanghai Jiao Tong University, Shanghai 200240, China \\ ${ }^{8}$ SUPA, Department of Physics, University of Strathclyde, Glasgow G4 ONG, United Kingdom
}

\begin{abstract}
By introducing a pre-plasma truncation for the cases with initial pre-plasma scale length larger than $0.2 \lambda$, the efficiency of high-order harmonics generated from relativistic laser-solid interaction can be enhanced by more than one order of magnitude and the angular spread can be confined into near-diffraction-limited divergence. Numerical simulations show that the density truncation results in more compact oscillation of the surface electron sheet and the curvature of the reflection surface for driving laser is greatly reduced. This leads to an overall improvement in the harmonic beam quality. More importantly, the density truncation makes the harmonic generation weakly dependent on the pre-plasma scale length, which provides a way to relax the extremely high requirement on the temporal contrast of the driving laser pulse. A feasible scheme to realize the required pre-plasma truncation is also proposed and demonstrated by numerical simulations.
\end{abstract}

\section{INTRODUCTION}

High-order harmonic generation (HHG) from ultraintense laser solid-target interaction provides an important way to produce bright coherent extreme-ultraviolet (XUV) and X-rays [1,2]. Owing to the high temporal and spatial coherence, high-order harmonics can generate attosecond pulses [3-8] and achieve diffraction-limited performance [9]. Due to its high brightness, the HHG source is very promising for applications in single-shot coherent diffraction imaging [10], X-ray-absorption fine-structure spectroscopy [11] and seeding free-electron lasers [12]. HHG is also proposed as an advanced tool for measuring magnetic fields and holograms on plasma surface [13-15], and for producing high-order vortex light $[16,17]$.

In the past decades, plenty of efforts have been devoted to improve the quality of HHG. To enhance the harmonic generation, the methods of using two-color lasers $[18,19]$, laser waveform shaping [20], multipass interactions [21] and using grating targets [22-24] were proposed. In particular, the pre-plasma scale length is found to be one of the key factors [25-28] for improving HHG efficiency. Harmonics generated around the front surface can be greatly enhanced when an optimal pre-plasma scale length $(0.05 \lambda<L<0.1 \lambda)$ is introduced [29], where $\lambda$ is the laser wavelength. However, ideal diffraction-limited

\footnotetext{
* minchen@sjtu.edu.cn

$\dagger$ zmsheng@sjtu.edu.cn
}

performance of harmonics can only be achieved by an ultra-steep steplike plasma surface. Vincenti et al. [30] demonstrated that the harmonic divergence could be enlarged by a factor of 3 for the optimal pre-plasma scale length, and even larger for longer pre-plasma scale length. In addition, ultrahigh laser contrast, $10^{-10} \sim 10^{-12}$, will be required if one expects to obtain the pre-plasma scale length as short as $0.05 \lambda \sim 0.1 \lambda[29,31]$. To overcome the above problems, a feasible method should be found to generate high-order harmonics with high efficiency and low divergence, and meanwhile suitably relax the severe requirement on the pre-plasma scale length.

Practically, the ramp shape of pre-plasma is also an important factor but has not been well explored. In the subrelativistic laser regime, Dromey et al. have found that by tuning the pre-plasma density ramp selected harmonics can be greatly enhanced [32]. However, this enhancement is limited to the harmonics generated by coherent wake emission mechanism [33], which will be out of action when the laser becomes relativistic. In the strongly relativistic laser regime, to our knowledge, the effects of pre-plasma ramp shape on HHG have not yet been reported.

In this paper, we will show that in the relativistic laser regime, for a target with relatively large pre-plasma length, a pre-plasma truncation within the overdense region can greatly enhance the HHG efficiency and obtain harmonics with near-diffraction-limited angular divergence. The HHG efficiency for the scale length $\mathrm{L}_{\dot{\mathrm{j}}} 0.2 \lambda$ after being truncated is comparable to that obtained for 
the theoretical optimal case with pre-plasma scale length of $\mathrm{L}=0.1 \lambda$. Our numerical studies show that when a relativistic laser impinges on a suitably truncated preplasma ramp, very compact and dense electron sheets can be driven to emit high-order harmonics. These electron sheets ultimately leading to significant enhancement of harmonic efficiency and reduction of spectral width. The truncation also makes it harder for the driving laser to dent the plasma surface. Thus, high-order harmonics can obtain near-diffraction-limited angular divergence. More importantly, in contrast to normally sensitive dependence of HHG on pre-plasma scale length, HHG under pre-plasma truncation is weakly dependent on the scale length, which partly relaxes the ultrahigh requirement on the laser temporal contrast for HHG. A feasible scheme to realize and control such pre-plasma truncation is also proposed.

\section{SCHEMATIC}

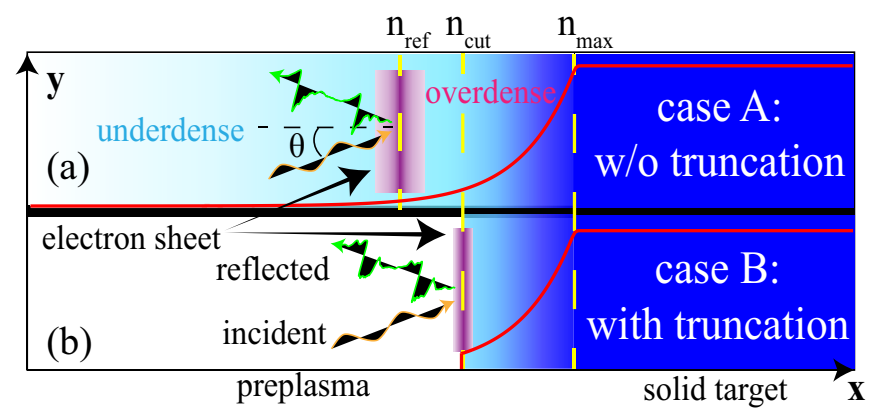

FIG. 1. Schematic of HHG in the laser-solid target interactions with different pre-plasma ramp shapes. The brown (green) arrows represent the incident (reflected) lasers. The purple layers represent the compressed electron sheets. The red lines show the plasma density distributions along the $\mathrm{x}$ direction. $n_{\text {ref }}, n_{\text {cut }}$ and $n_{\max }$ denote the reflection density, the truncation density and the maximum plasma density of solid target, respectively.

The main idea is illustrated in Fig. 1. When a short relativistic laser pulse irradiates a solid target with normal pre-plasma profile as shown in Fig. 1(a), electrons in the pre-plasma are firstly compressed and pushed forward by the laser. The charge separation between the compressed electrons and immobile ions builds an electrostatic restoring force which pulls the electron sheet back. Thus, an electron sheet acting as "oscillating mirror" is formed and oscillates around the laser reflection region. When the incident laser is reflected by the back-moving electron sheet, its frequency is upshifted due to the Doppler effect and high-order harmonic components appear in the reflected pulse [34, 35]. For a linearly polarized laser with normalized intensity of $a_{0}=e E / m_{e} \omega_{0} c$ and incidence angle of $\theta$, it will be reflected around the region with density $n_{\text {ref }}=\gamma n_{c} \cos ^{2} \theta[36]$, where $\gamma=\left(1+a_{0}^{2} / 2\right)^{1 / 2}$ is the relativistic factor of the electrons around there and $n_{c}=\epsilon_{0} m_{e} \omega_{0}^{2} / e^{2}$ represents the non-relativistic critical plasma density. However, if the pre-plasma scale length is too large, the laser pulse would pass through a longdistance of underdense pre-plasma before arriving at the reflection region. The relatively low plasma density leads to a long pile-up length of electrons to build the restoring force. Furthermore, the laser propagation in the nearcritical-density plasma before reflection may give rise to uncontrolled laser evolution and pre-heated electrons. As a result, the compression of the electron sheet and the coherence of HHG are reduced. Besides, the reflection surface will be curved by the driving laser at low plasma density region. Since the angular divergence of high-order harmonics could be enlarged due to the curved surface, the beam quality will then be largely reduced. In order to improve the HHG quality, a reasonable way is to push the oscillation position deeper into a higher density region. As shown in Fig. 1(b), if the pre-plasma is truncated at a position with density $n_{\text {cut }}>n_{\text {ref }}$, a more compact electron sheet could be generated by the laser within a short compression distance. As we will see, the subsequent HHG quality in this scheme can be greatly improved compared with the HHG from a target with continuous pre-plasma.

\section{EFFECTS OF PRE-PLASMA TRUNCATION ON HHG}

We use two-dimensional particle-in-cell (PIC) simulations to study the harmonic generation. A p-polarized laser pulse with beam waist of $w=3 \lambda_{0}$ and duration of $\tau=10 T_{0}$, is incident from the left boundary of the simulation box, where $\lambda_{0}=800 \mathrm{~nm}$ and $T_{0}=\lambda_{0} / \mathrm{c}$ are the laser wavelength and period, respectively. The normalized laser intensity is $a_{0}=3$ and the incidence angle is $\theta=45^{\circ}$. These parameters can be realized by usual 100TW Ti:sapphire femtosecond laser. The target consists of a $2 \lambda_{0}$ thick slab with $n_{\max }=100 n_{c}$ and an exponentially declining pre-plasma with a scale length of $L$. The grid size is $d x=d y=\lambda_{0} / 200$, and 100 particles per cell are used in the simulations.

\section{A. Harmonic Spectrum}

We first compare the harmonic spectra of three cases, $L=0.1 \lambda$ without pre-plasma truncation (case $\mathrm{S}$, corresponding to an optimal scale length), $L=0.2 \lambda$ without truncation (case A, corresponding to a usual experimental condition) and $L=0.2 \lambda$ with a truncation at density of $n_{\text {cut }}=7 n_{c}$ (case B). The simulated harmonic spectra are shown in Fig. 2. One can see that the harmonic efficiency decreases by near one order of magnitude from $L=0.1 \lambda$ to $L=0.2 \lambda$ if without pre-plasma truncation. This phenomenon is a consensus in simulations and experiments $[28,29]$. However when pre-plasma truncation 
is applied (case B), the harmonic intensity recovers to a high level and is comparable to that in case S. The enhanced harmonic spectrum by truncation has more interesting features. Harmonics with order $10<N<30$ (XUV) are enhanced by more than one order of magnitude in their efficiency despite the spectrum declines faster at higher orders. In addition, the spectral width of each harmonic is narrower compared with those in case A, which can be seen more clearly in the enlarged illustration of Fig. 2. Besides, the envelope of harmonic spectrum exhibits regular modulation.

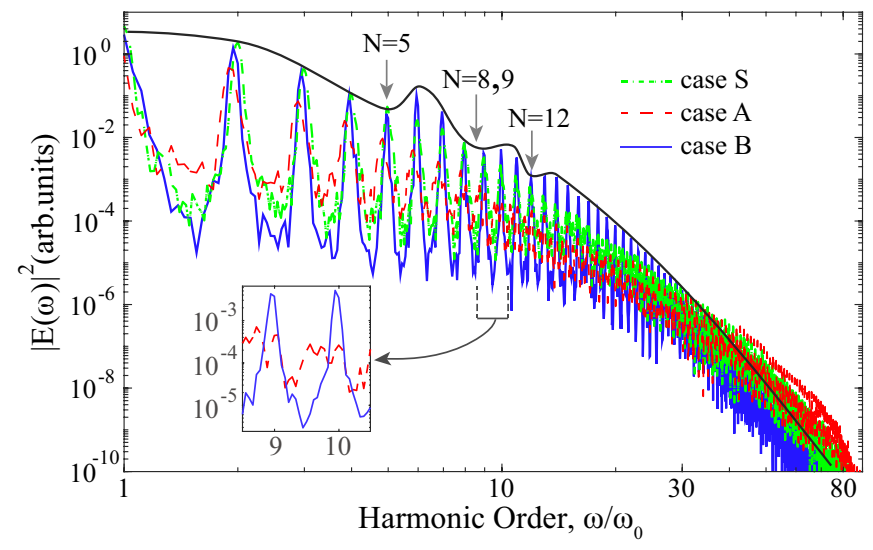

FIG. 2. Harmonic spectra for the cases $L=0.1 \lambda$ (case $\mathrm{S}$, dashed dotted line), $L=0.2 \lambda$ without pre-plasma truncation (case A, dashed line) and $L=0.2 \lambda$ with truncation at $n_{\text {cut }}=$ $7 n_{c}$ (case B, solid line).

To understand these new features and the reason for the recovery of harmonic intensity, we first compare the dynamics of surface electrons and consequent HHG for cases A and B. Figs. 3(a) and (b) show the spatiotemporal density evolution of electrons along a fixed line $\left(-1.5 \lambda_{0}<x<0, y=0\right)$. One can see that the electron sheet in case A mainly oscillates with laser frequency $\omega_{0}$ and has a rather long excursion distance. However, in case $\mathrm{B}$, the electron sheet is much more compact and it oscillates twice per laser cycle. The excursion distance is also shorter than that in case A. These imply that the coherent superposition of the harmonic emission from this electron sheet could be improved and the radiation may occur twice within one laser cycle. In Figs. 3(c) and (d) the typical electron trajectories and the concomitant harmonic emission demonstrate these conjectures.

The oscillation mode of electron sheet is mainly determined by the laser fields at the reflection surface. For case A, the laser energy will be strongly absorbed around the near-critical-density region. Because of the short scale length of pre-plasma, the so-called "vacuum heating" [37] takes main effects on the HHG process. The reflection laser is weaker than the incident one. Therefore, electron sheets oscillate resonantly with the longitudinal electric field of laser, once per laser cycle. However, for case B, the laser will suffer from near-complete reflection at the surface due to the high plasma density. The
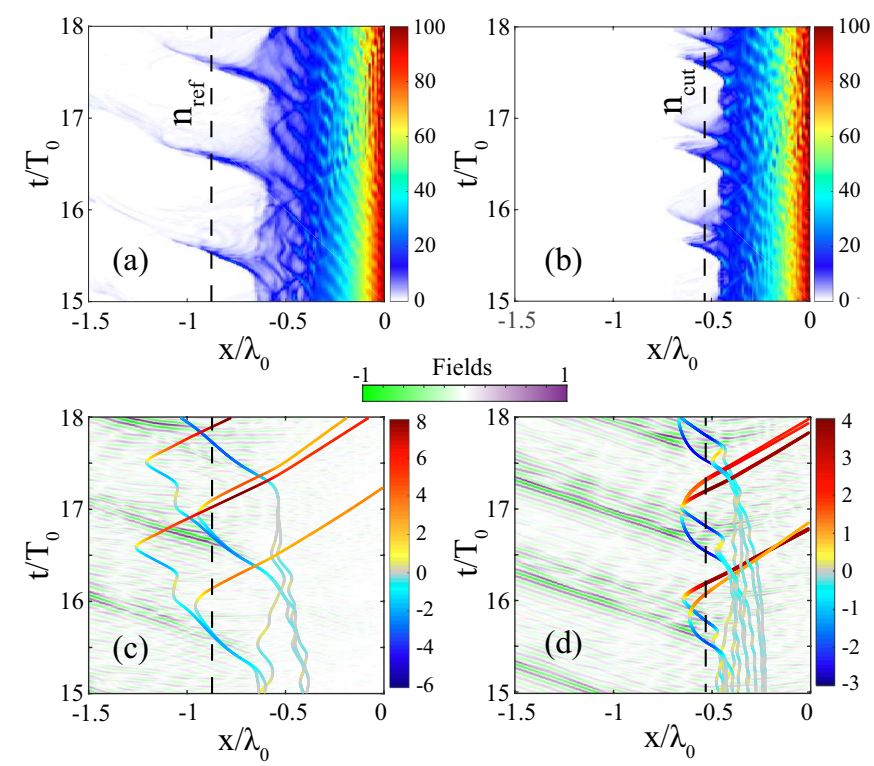

FIG. 3. (a) and (b): Spatio-temporal evolution of electron density along a fixed line with $y=0$. (c) and (d): Typical trajectories of the electrons composing the oscillating mirror. The background shows harmonic emission filtered by $N \geq 10 t h$ (top colorbar). The colors along the trajectories represent the normalized longitudinal momentum $p_{x}=\gamma v_{x} / c$ of the electrons (right colorbar). The left column [(a) and (c)] and right column [(b) and (d)] figures correspond to the cases without and with $n_{\text {cut }}=7 n_{c}$, respectively.

longitudinal electric field will be counteracted by the incident and reflected lasers. The residual fields are the transverse electric field $E_{y}=2 E_{0} \sin \theta$ and magnetic field $B_{z}=2 B_{0}$. In this scenario, the oscillation of surface electrons is dominated by the magnetic field - " AC gyromagnetic effects" [38]. As demonstrated in Ref.[38], the $A C$ gyromagnetic effects will inhibit the longitudinal acceleration of surface electrons and change their oscillation frequency. The oscillation frequency should ideally be $\omega_{B}=2 \cos \theta a_{0} \omega_{0} \approx 4.2 \omega_{0}$ for our simulation parameters. However, the real oscillation frequency from Fig. 3(d) is found to be $\omega_{B}=3.4 \omega_{0}$. Two reasons may lead to this discrepancy. First, the laser is actually not completely reflected in our case B. A fraction of laser energy is absorbed by surface electrons, which reduces the intensity of the reflected laser. Second, some electrons driven by the laser fields are still strongly relativistic. As shown in Fig. 3(d), these energetic electrons will ultimately move into the target and not participate in the next oscillation, which affects the surface fields.

After understanding the oscillation mode at different cases, a clear explanation of the spectral enhancement and modulation for case B can be given. We select a stripe of electrons around the laser focus with a transverse width of $\delta y=0.05 \lambda_{0}$. Their relative density distribution in the phase space $\left(x-4 \gamma^{2}\right)$ at the moment when the oscillation velocity of compressed electron sheet reaches its maximum are shown in Fig. 4(a). As one can 

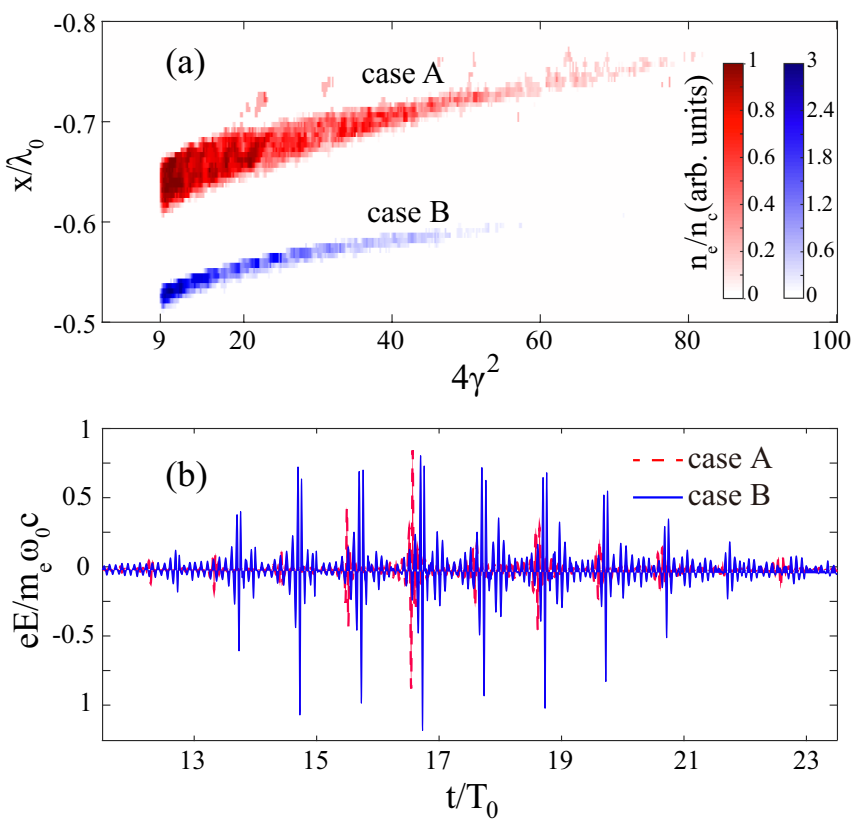

FIG. 4. (a) Relative electron density distribution in the phase space $n_{e}\left(x, 4 \gamma^{2}\right)$. Only electrons with $\gamma>1.5$ are counted. (b) Temporal waveform of harmonic fields filtered by $N \geq 10 t h$. The peak of driving laser reaches the target surface at about $t=17 T_{0}$.

see, the electron sheet in case B is much thinner and the density is much higher than those in case A. The smaller bunch size and higher density are favorable for coherent and stronger radiation. On the other hand, there are fewer electrons in the high-energy tail in case B. Since the maximum radiation frequency is limited by the electron relativistic parameter $\gamma$, the harmonic spectrum in the higher order region $(N>30)$ declines faster in case $\mathrm{B}$ than in case $\mathrm{A}$, as shown in Fig. 2. The inhibition of high-energy electrons in case B is due to the aforesaid gyromagnetic effects. Such inhibition will be stronger with the increase of $n_{\text {cut }}$ because of the increased laser reflectivity and surface current to enhance magnetic fields. Therefore, there should be an optimal truncation density, as it will be shown in Sec. IV, which is most suitable for enhancing the middle-order harmonics, i. e. in the XUV range.

We then analyze the spectral modulation. As shown in Fig. 4(b), high-order harmonics are not emitted efficiently every laser cycle for case A. Only around the peak temporal position when the plasma surface is compressed to be denser the harmonic intensity is comparable to the intensity for case B. In case B, the HHG is always efficient due to the plasma surface is initially dense. For each laser cycle, the radiated harmonic signal has a similar temporal profile we define as $f(t)$, then the final spectrum is $E(\omega)=\Sigma_{m=0}^{M-1} F(\omega) e^{-i m \omega T_{0}}$, where $M$ is the number of effective laser period for HHG and $F(\omega)$ is the Fourier transformation of $f(t)$. The spectral envelope is determined by $F(\omega)$ and the harmonic peaks come from the coherent addition term $\exp \left(-i m \omega T_{0}\right)$. Each peak width is inversely proportional to $M$. This is why the spectral width of each harmonic is narrower for case B. In addition, if for a case, just like case B, there are twice similar emissions per laser cycle, i.e. $f(t)=f_{0}(t)+\alpha f_{0}(t+\Delta t)$, then $F(\omega)=F_{0}(\omega)[1+\alpha \exp (i \omega \Delta t)]$. This leads to the spectral modulation and its intensity will be weakened at $\omega \Delta t=(2 p+1) \pi$, where $p=1,2,3, \cdots$. Hence, the modulated orders are at $N=(p+1 / 2) T_{0} / \Delta t$. In case $\mathrm{B}$, the temporal interval of emission is approximately $T_{0} / \Delta t=\omega_{B} / \omega_{0}=3.4$ as shown in Fig. 3(d). So the calculated modulation orders are $5,8.5,12, \cdots$, which is in good agreement with the simulation results shown in Fig. 2.

\section{B. Harmonic angular divergence}

Besides the spectral intensity and width, the angular divergence of harmonic beam is also important for applications. In our studies, we find the pre-plasma truncation can remarkably reduce the harmonic angular divergence. Figure 5 shows the curvature of plasma surface for case $\mathrm{A}$ and $\mathrm{B}$. When the pre-plasma is not truncated, the maximum bending distance can reach $\delta x_{e}=0.4 \lambda_{0}$ even though $L=0.2 \lambda_{0}$. However, the bending distance is reduced to $\delta x_{e}=0.08 \lambda_{0}$ after pre-plasma truncation. According to the analyses in Ref. [30], the curved surface acts as a focusing mirror which focuses the high-order harmonics with a divergence angle $\theta_{N}=\theta_{N}^{0} \sqrt{1+\Psi_{N}^{2}}$, where $\theta_{N}^{0}=\lambda_{N} /\left(\pi w_{N}\right)$ is the diffraction-limited divergence angle of the $N^{\text {th }}$ harmonic without surface curvature, and $\Psi_{N}=\pi w_{N}^{2} /\left(r_{d} \lambda_{N} \cos \theta\right)$ is the dimensionless focusing parameter for the $N^{t h}$ harmonic. $r_{d}$ is the curvature radius of plasma surface. If $\Psi_{N} \ll 1$, the harmonic divergence angle is $\theta_{N} \approx \theta_{N}^{0}$, decreasing near linearly with $N^{-1}$. While if $\Psi \gg 1$, the harmonic divergence angle is $\theta_{N} \approx \Psi_{N} \theta_{N}^{0}=w_{N} /\left(r_{d} \cos \theta\right)$, which is weakly dependent on the harmonic order because $w_{N}$ changes slowly with $N$.

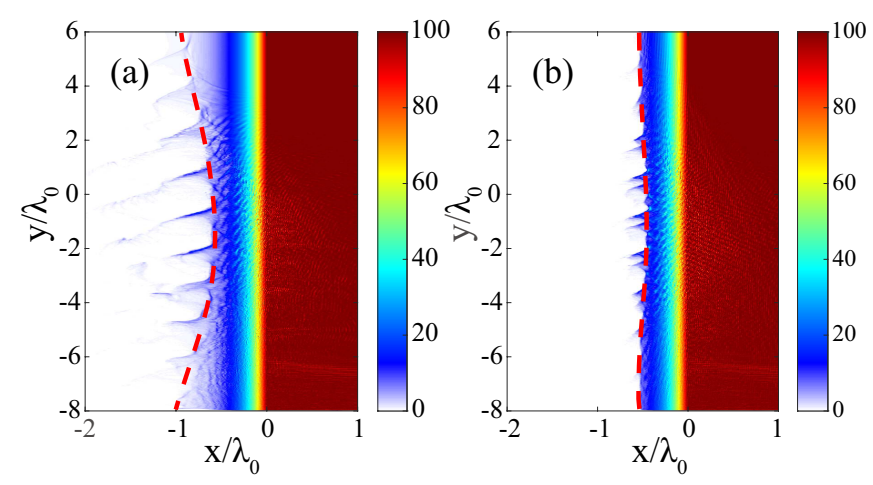

FIG. 5. Plasma density $n_{e}$ profiles for cases without preplasma truncation (a) and with $n_{\text {cut }}=7 n_{c}$ (b) at $t=17 T_{0}$. The red lines shows the outlines of plasma surface at different cases. 
In our simulations, case $\mathrm{A}$ and case $\mathrm{B}$ correspond to $\Psi \gg 1$ and $\Psi<1$, respectively. As shown in Fig. 6, the divergence angle of each harmonic almost keeps a constant of $120 \mathrm{mrad}$ for case A, however for case B the divergence angle decreases linearly with $N^{-1}$ as $\theta_{N}=420 / N$ mrad when $N \geq 10$. We note that the simulated divergence angles are smaller than the estimates by the model in Ref. [30]. In Fig. 5, the curvature radius is $r_{d} \approx w_{0}^{2} / 2 \delta x_{e}=11 \lambda_{0}$ for case A. One can calculate out $\Psi \gg 1$ for all high-order harmonics and the divergence angle should be $\theta_{N} \approx 380 \mathrm{mrad}$. The reason for the divergence reduction can be found from Figs. 3(c) and (d). It can be seen that the emission of harmonics does not occur at the end of curvature surface. High-order harmonics are emitted by the energetic electron beams after a distance of acceleration. Thus, the real curvature for focusing harmonics is less than the curvature shown in Fig. 5. Particularly, for the truncation case B, only those electrons getting back to the initial surface could obtain enough energy to emit higher-order harmonics due to the gyromagnetic effects, as shown in Fig. 3(d). Hence, the plasma surface seems hardly curved and can produce high-order harmonics with near-diffraction-limited divergence.
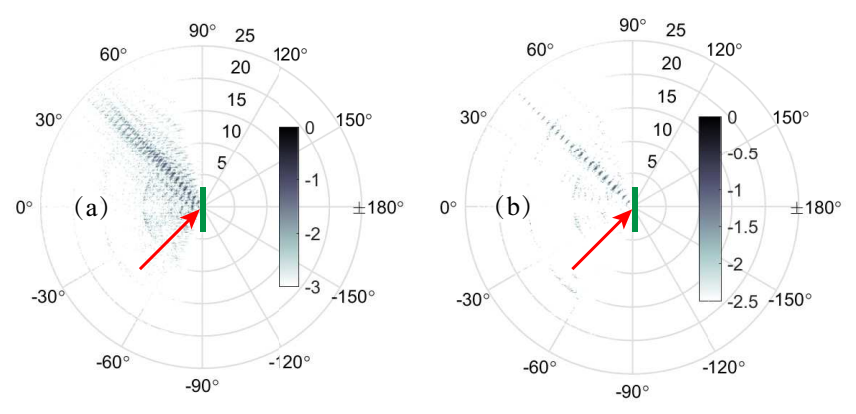

FIG. 6. Angular divergence of harmonics for cases without pre-plasma truncation (a) and with $n_{c u t}=7 n_{c}$ (b). The radial dimension represents the harmonic order. The green rectangles represent the targets, and the red arrows represent the incident laser. The colorbars show the logarithm of normalized harmonic intensity.

\section{HHG dependence on pre-plasma scale length}

As shown in Fig. 3, the oscillating electron sheet is more compact in the truncation case. The maximum excursion distance of oscillating electron sheet can be given by $\delta x_{e}=L \ln \left[1+\lambda_{0} a_{0}(1+\sin \theta) n_{c} /\left(\pi L n_{\text {cut }}\right)\right]$ [30]. If $n_{\text {cut }}$ or $L$ is large enough to make $n_{\text {cut }} / n_{c} \gg$ $\lambda_{0} a_{0}(1+\sin \theta) / \pi L, \delta x_{e}$ will be simplified as $\delta x_{e}=$ $\lambda_{0} a_{0}(1+\sin \theta) n_{c} / \pi n_{c u t}$, which is independent of the preplasma scale length. The value of $n_{\text {cut }}$ could be a little smaller than the above estimation due to the real excursion distance, where high-order harmonics are produced, is smaller than $\delta x_{e}$.
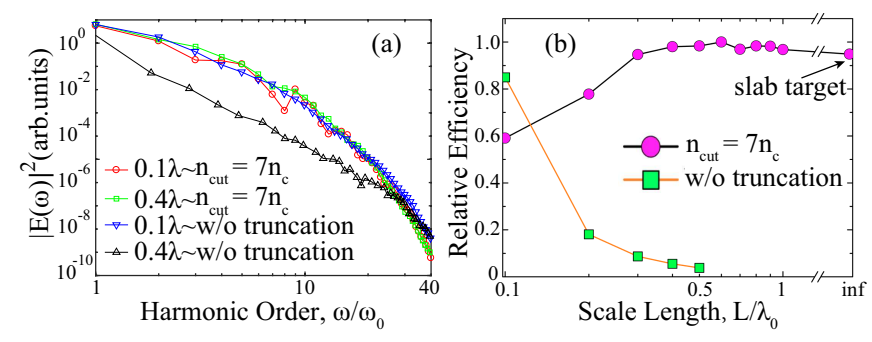

FIG. 7. (a) Harmonic spectra (only peak points shown) for the pre-plasma scale lengths of $L=0.1 \lambda_{0}$ and $0.4 \lambda_{0}$, with $n_{\text {cut }}=7 n_{c}$ and without truncation. (b) Relative efficiency of harmonic yield $\left(\sum_{N \geq 2}\left|E\left(\omega_{N}\right)\right|^{2}\right)$ for cases with different plasma scale lengths.

Our studies show that HHG with pre-plasma truncation has the advantage of being insensitive to the preplasma scale length. Figure 7(a) shows the harmonic spectra for four cases. For the cases without pre-plasma truncation, harmonic intensity drops more than two orders of magnitude as the pre-plasma scale length increases from $L=0.1 \lambda_{0}$ to $0.4 \lambda_{0}$. On the contrary, the harmonic spectra for the cases with truncation do not change much as the pre-plasma scale length varies. Harmonic yields for cases with and without pre-plasma truncation at different plasma scale lengths are shown in Fig. 5(b). The generation efficiency reduces rapidly as the plasma scale length increases in the cases without truncation. When $L>0.5 \lambda_{0}$, almost no harmonics can be generated. However, the efficiency always remains at a high level when the pre-plasma is truncated with $n_{c u t}=7 n_{c}$. It is worth noting that although HHG efficiency can be improved by tuning the pre-plasma scale length, as shown in Fig. 7(a), and the scale length can be controlled by improving the laser contrast using plasma mirror technique [31], usually more than $30 \%$ of the laser energy is inevitably lost. The insensitive dependence of HHG on pre-plasma scale length can relax the requirement on ultrahigh laser contrast.

\section{OPTIMAL TRUNCATION DENSITY}

According to our analyses in Sec.III, using pre-plasma truncation can improve the radiation coherence of harmonic beams, however higher truncation density will inhibit the generation of high-energy electrons. To come to a compromise, there should be an optimal truncation density which leads to the most efficient emission of middle-order harmonics, i. e. the XUV light. To investigate the optimal truncation density for HHG, we performed a series of simulations by scanning $n_{\text {cut }}, a_{0}$ and $\theta$.

Figure $8(\mathrm{a})$ shows the variation of harmonic intensities with different $n_{\text {cut }}$. It shows that when $n_{\text {cut }}$ is below the non-relativistic critical density $\left(n_{c} \cos ^{2} \theta\right)$, the truncation density has very weak influences on the har- 

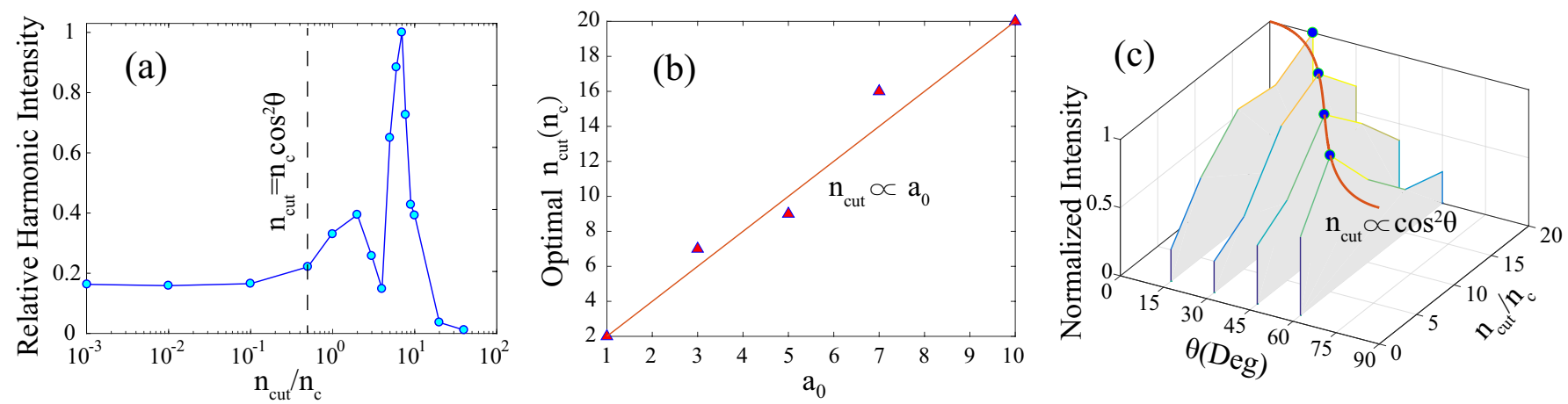

FIG. 8. (a) Dependence of harmonic intensity $\left(\sum_{N \geq 10}\left|E\left(\omega_{N}\right)\right|^{2}\right)$ on $n_{\text {cut }}$ for $a_{0}=3$. (b) Optimal truncation density $n_{\text {cut }}$ for HHG varying with laser intensity. The laser incidence angle in (a) and (b) is $\theta=45^{\circ}$. (c) Dependence of harmonic intensity on $\theta$ and $n_{\text {cut }}$. The laser intensity in (c) is $a_{0}=5$.

monic intensity. However, when the truncation density increases, the influence becomes effective. The HHG becomes quite efficient when $n_{\text {cut }}$ is close to $n_{\text {ref }}$, around which harmonic emission reaches a strong point and is emitted once per laser cycle. Nevertheless, the harmonic intensity becomes even higher when the truncation density increases to $n_{c u t}=7 n_{c}$ where the emission occurs twice per laser cycle as we showed before. The optimal truncation density also relies on the laser intensity $a_{0}$ and incidence angle $\theta$. In Fig. 8(b) we show the optimal truncation densities for different laser intensities when the incidence angle is fixed to be $\theta=45^{\circ}$. It shows that the optimal truncation density is proportional to the laser intensity. In Fig. 8(c) we show the harmonic intensity varying with $\theta$ and $n_{\text {cut }}$. It can be found that the optimal truncation density is proportional to $\cos ^{2} \theta$. In general, our simulations show that the optimal truncation density is proportional to the multiple of the laser intensity and the relativistic critical density, which approximately equals $n_{\text {cut }} \approx 4 a_{0} n_{c} \cos ^{2} \theta$. This result is consistent with the simulation results in Refs. [39] and [40], where HHG is found to be most efficient when the self-similarity parameter $S=n_{e} / a_{0} n_{c}$ of surface plasma is in the range $1 / 2<S<4$.

\section{PRE-PLASMA TRUNCATION REALIZATION}

We have illustrated that the beam quality-radiation efficiency and angular divergence - of high-order harmonics can be improved by truncating plasma at an optimized density position. The main reason why HHG experiments with an optimized $S$ have not been carried out so far is related to the great difficulty of producing plasma with appropriate densities. The scheme proposed by us could provide a solution to this problem. In practice, if the target plasma density is initially as low as the optimal truncation density (like foam targets), high HHG efficiency seems likely to be obtained directly and thus truncation is not necessary. However, the sur- faces of such targets are hard to be smooth enough to fulfill the HHG requirement [9]. For this reason, HHG experiments are usually carried out by using the polished fused silica glass, whose plasma density is about $400 n_{c}$ when being fully ionized. To effectively produce high-order harmonics from glass targets, a certain size of pre-plasma $(L<0.1 \lambda)$ needs to be created. Under such short scale lengths, pre-plasma truncation is also not necessary to improve the HHG efficiency, as shown in Fig. 7(b). Nevertheless, the truncation will be effective for HHG when the pre-plasma scale length is relatively larger in experiments. Besides, as we mentioned before, near-diffraction-limited harmonic beams are easier to be obtained by pre-plasma truncation.

Here we propose a feasible scheme to realize the required pre-plasma truncation. The parameters of our scheme are based on the 200TW Ti:sapphire laser system at the Laboratory for Laser Plasmas of Shanghai Jiao Tong University [41]. As illustrated in Fig. 9(a), a truncation pulse (TP) is cut from the main laser beam (MP) and it is then converted to circular polarization by a $1 / 4$ waveplate. This TP is normally focused on the solid target surface using an off-axis parabolic (OAP) mirror with long focal length. The MP is obliquely focused on the target using an OAP with short focal length. By this configuration, the TP has a larger focal spot than the MP; thus the modulated pre-plasma by the TP can be viewed as a transversely plane structure for the MP.

The TP is designed to be picoseconds ahead of the MP. Two-dimensional PIC simulation demonstrates that transversely flat, longitudinally steep pre-plasma truncation can be obtained during this time interval. Fig. 9(b) shows the typical distributions of ion charge density at the beginning and $t=2$ ps. Initially, the plasma parameters are the same as the proposed parameters in Sec.III, but the ions $\left(\mathrm{O}^{8+}\right)$ are mobile here. The normalized laser intensity of TP is $a_{0}=0.3$ and the beam waist is $w_{0}=6 \lambda_{0}$. The TP irradiates on the targets at $t=0$. After 2 ps evolution, it can be clearly seen that the pre-plasma is steeply truncated along the longitudinal direction. Along the transverse direction, there is a flat 
(a)

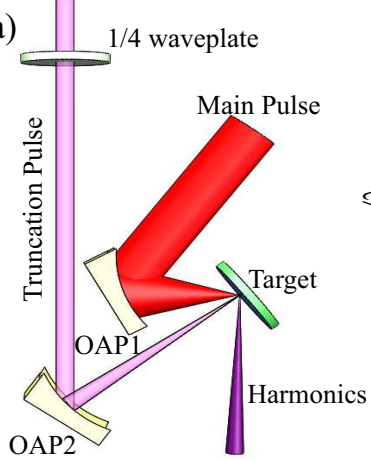

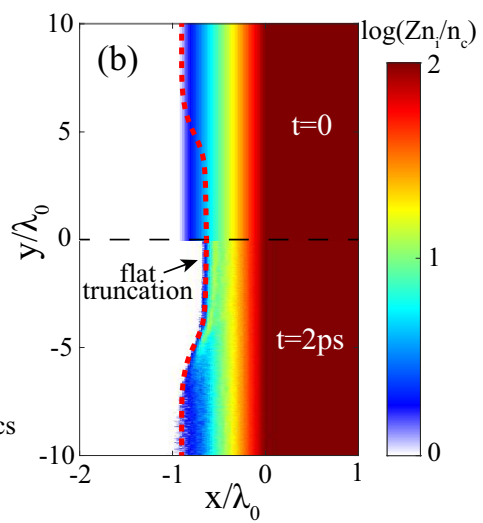

FIG. 9. (a) A feasible experimental scheme for high-quality HHG by preplasma truncation. The violet beam represents the truncation pulse with circular polarization. It normally irradiates on the solid target and generates a steplike profile in the preplasma. The red beam represents the main laser pulse. It excites the relativistic oscillation of truncated plasma surface and then generates harmonics. (b) Ion charge density distributions at different instants. The top half shows the initial density distribution with pre-plasma scale length $L=0.2 \lambda$. The bottom half shows the truncated density distribution after being compressed by a circularly polarized laser with $a_{0}=0.3, w_{0}=6 \lambda_{0}$, and $\tau=10 T_{0}$. The red line shows the outline of plasma surface at $t=2 \mathrm{ps}$.

region with a width of $\sim 6 \lambda_{0}$. This truncation structure is close to the schematic illustrated in Fig. 1(b).

The reason for the generation of pre-plasma truncation by a normally incident, circularly polarized laser is as follows. When the circularly polarized TP irradiates on the pre-plasma, its energy is barely absorbed. Assuming the $\mathrm{TP}$ is completely reflected at the critical density surface, the momentum flux conservation gives $2 I / c=n_{c} p_{x}^{2} / m_{e}$, where $I$ is the laser intensity. Surface electrons will be pushed forward by this radiation pressure. In the simulation we found after the laser irradiance the increments of the electron transverse momenta are almost zero and the electrons are inefficiently heated. The kinetic energy of surface electrons $E_{k}=\left(\sqrt{1+p_{x}^{2} c^{2} / m_{e}^{2} c^{4}}-1\right) m_{e} c^{2}$ could be rewritten as $E_{k}=\left[\sqrt{1+2 I /\left(n_{c} c \cdot m_{e} c^{2}\right)}-1\right] m_{e} c^{2}$. Due to the collective motion of these electrons, very strong electric fields will be built in a rather short distance and drag the ions to move together with electrons. When $I=2 \times 10^{17} \mathrm{~W} / \mathrm{cm}^{2}\left(a_{0}=0.3\right)$, the kinetic energy of surface electrons is $E_{k} \approx 20 \mathrm{keV}$. The static electric field will drag the ions to move with these electrons in a distance of $0.03 \lambda_{0}$. After the TP irradiation, the surface plasma will move forward and pile up gradually. By this way, a steplike structure is formed in the front of preplasma. To maintain the steplike structure, the uncompressed pre-plasma should be relatively cold. It can be inferred that the steplike structure will collapse faster if the background temperature of pre-plasma is higher. Besides, the oblique incidence and linear polarization of TP are not recommended because they will produce plenty of

hot electrons. These hot electrons are detrimental for the longitudinal compression and may give rise to transverse instability, like Weibel instability [42].
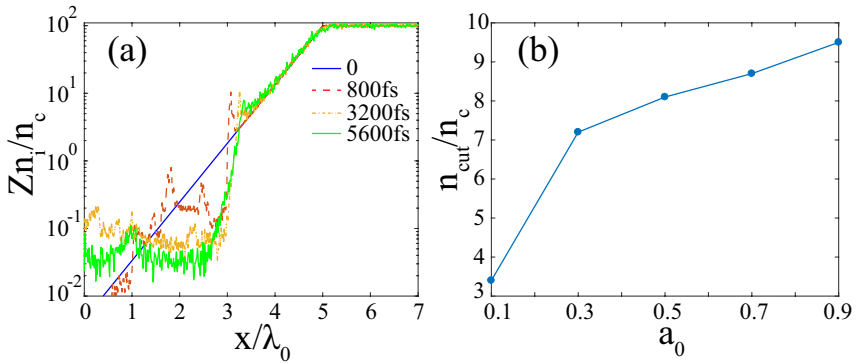

FIG. 10. (a) Ion charge density profiles at different instants after being compressed by a circularly polarized laser. The initial pre-plasma scale length is $L=0.5 \lambda_{0}$. (b) Effective truncation density for truncation pulse with different intensities.

We further performed one-dimensional PIC simulations to make clear how the pre-plasma evolves in the compression process. The pre-plasma scale length is set to be $L=0.5 \lambda_{0}$. This scale length is suitable for a fused silica glass irradiated by a laser with $10^{-9}$ contrast [41], where the target surface would be preheated by the $10 \mathrm{ps}$-ahead laser pedestal to a $10 \mathrm{eV}$-level temperature. The $10^{-9}$ contrast is currently a standard configuration for the commercial hundreds-of-terawatts laser chains. In the one-dimensional simulations, the grid size is $d x=\lambda_{0} / 1000$ and each grid cell is filled with 144 macro electrons and ions. The numerical heating in these simulations is strictly inhibited. The normalized TP laser intensity is also $a_{0}=0.3$ and the laser duration is $\tau=10 T_{0}$. Figure 10(a) shows the ion charge density profiles at different instants when the plasma temperature is initially $10 \mathrm{eV}$. It can be seen that very steep pre-plasma truncation can be built within one picosecond. However, at this time some soliton structure in the near-critical-density region is also formed [43] and will influence the propagation of main pulse. The soliton structure will be dissipated soon. After two picoseconds, the pre-plasma ramp shape tends to be stable. As shown in Fig. 10(a), the steplike structure can maintain for several picoseconds, which gives a large time window for the main pulse to interact with the truncated plasma. The fluid velocity of the truncated surface is $v_{\text {fluid }} \sim 10^{-4} c$, which will hardly influence the HHG process. Besides, the low-density plasma $\left(n_{e} \ll 1 / 4 n_{c}\right)$ formed in front of the truncation has little influence on HHG, which has been confirmed in Fig. 8(a). We also test the feasibility of pre-plasma truncation at higher background temperature. It is shown that when the initial plasma temperature is $100 \mathrm{eV}$, the steplike structure can only maintain for less than 1ps and then evolves into a short-scale pre-plasma; when the temperature increases to $1 \mathrm{keV}$, no truncation can be found in the whole simulation.

Finally, the effective truncation density changing with 
TP intensity is shown in Fig. 10(b). The effective truncation density represents the time-averaged $n_{\text {cut }}$ before the steplike structure collapses. It can be seen that $n_{\text {cut }}$ increases approximately linearly when $0.3 \leq a_{0} \leq 0.9$.

\section{CONCLUSION}

In summary, we have shown that for large pre-plasma scale length, the beam quality of high-order harmonics generated from relativistic laser-solid target interaction can be greatly improved through pre-plasma truncation. Compared to the cases with pre-plasma scale length $L>0.2 \lambda_{0}$, harmonic intensity can be enhanced by more than one order of magnitude after truncation. Harmonic spectrum has narrower spectral width and regular modulation. The angular spread of harmonic beam is well confined into near-diffraction-limited divergence.
Besides, when the pre-plasma truncation is used, the harmonic yield weakly depends on the pre-plasma scale length. This advantage can partly relax the ultrahigh requirement on laser contrast in HHG experiments. By numerical simulations, the optimal truncation density is found to be $n_{\text {cut }} \approx 4 a_{0} n_{c} \cos ^{2} \theta$. Such truncation can be realized by tailoring pre-plasma with another normally incident, circularly polarized laser pulse. We expect our scheme could provide a useful way to obtain high-order harmonics on the laser facilities of hundreds of terawatts.

This work was supported by the National Natural Science Foundation of China (11774227, 11721091,11905129), Science Challenge Project (TZ2018005) and China Postdoctoral Science Foundation (2017M621443). The authors thank ПI Supercomputer center at SJTU and SR acknowledges the usage of the Skoltech CDISE HPC cluster Zhores [44] for performing simulation studies. The authors also appreciate the referees' very helpful comments.
[1] P. Gibbon et al. Phys. Rev. Lett. 76, 50 (1996).

[2] U. Teubner et al. Rev. Mod. Phys. 81, 445 (2009).

[3] S. G. Rykovanov et al. New J. Phys. 10, 025025 (2008).

[4] P. Heissler et al. Phys. Rev. Lett. 108, 235003 (2012).

[5] H. Vincenti et al. Phys. Rev. Lett. 108, 113904 (2012).

[6] Y. Nomura et al. Nat. Phys. 5, 124 (2009).

[7] J.A. Wheeler et al. Nat. Photon. 6, 829 (2012).

[8] J.M. Mikhailova et al. Phys. Rev. Lett. 109, 245005 (2012).

[9] B. Dromey et al. Nat. Phys. 5, 146-152 (2009).

[10] A. Ravasio et al. Phys. Rev. Lett. 103, 028104 (2009).

[11] D. Popmintchev et al. Phys. Rev. Lett. 120, 093002 (2018).

[12] G. Lambert et al. Nat. Phys. 4, 296-300 (2008).

[13] I. Watts et al. Phys. Rev. Lett. 88, 155001 (2002).

[14] U. Wagner et al. Phys. Rev. E 70, 025401 (2004).

[15] A. Leblanc et al. Nat. Phys. 13, 440-443 (2017).

[16] X. M. Zhang et al. Phys. Rev. Lett. 114, 173901 (2015).

[17] A. Denoeud et al. Phys. Rev. Lett. 118, 033902 (2017).

[18] M. R. Edwards et al. Opt. Lett. 39, 6823-6826 (2014).

[19] M. Yeung et al. Nat. Photon. 11, 32-35 (2017).

[20] M. R. Edwards et al. Phys. Rev. Lett. 117, 125001 (2016).

[21] M. R. Edwards et al. Phys. Rev. A 93, 023836 (2016).
[22] X. Lavocat-Dubuis et al. Phys. Rev. E 80, 055401 (2009).

[23] G. Zhang et al. Opt. Express 25, 023567 (2017).

[24] G. Cantono et al. Phys. Rev. Lett. 120, 264803 (2018).

[25] F. Dollar et al. Phys. Rev. Lett. 110, 175002 (2013).

[26] C. Roedel, et al. Phys. Rev. Lett. 109, 125002 (2012).

[27] M. Bocoum, et al. Phys. Rev. Lett. 116, 185001 (2016).

[28] L. Chopineau, et al. Phys. Rev. X 9, 011050 (2019).

[29] S. Kahaly et al. Phys. Rev. Lett. 110, 175001 (2013).

[30] H. Vincenti et al. Nat. Commun. 5, 3403 (2014).

[31] C. Thaury et al. Nat. Phys. 3, 424-429 (2007).

[32] B. Dromey et al. Phys. Rev. Lett. 102, 225002 (2009).

[33] F. Quere et al. Phys. Rev. Lett. 96, 125004 (2006).

[34] S. V. Bulanov et al. Phys. Plasmas 1, 745 (1994).

[35] R. Lichters et al. Phys. Plasmas 3, 3425 (1996).

[36] W. L. Kruer, The physics of laser plasma interactions, Addison-Wesley, Reading, MA, 1988.

[37] F. Brunel Phys. Rev. Lett. 59, 52 (1987).

[38] J. P. Geindre et al. Phys. Rev. Lett. 97, 085001 (2006).

[39] A. A. Gonoskov et al. Phys. Rev. E 84, 046403 (2011).

[40] T. G. Blackburn et al. Phys. Rev. A 98, 023421 (2018).

[41] J. Gao et al. Phys. Plasmas 103, 103102 (2019).

[42] L. O. Silva et al. Phys. Plasmas 9, 2458 (2002).

[43] Y. Sentoku et al. Phys. Rev. Lett. 83, 3434 (1999).

[44] I. Zacharov et al. arXiv: 1902.07490 (2019). 